\title{
LETTER
}

\section{Contralateral breast cancer risk in BRCA1/2-positive families needs to be adjusted for phenocopy rates particularly in second-degree untested relatives}

\author{
D Gareth R Evans ${ }^{* 1,2}$, Anthony Howell', Sarah L Ingham ${ }^{1,3}$ and lain Buchan ${ }^{3}$ \\ See related research by Rhiem et al., http://breast-cancer-research.com/content/14/6/R156
}

In the previous issue of Breast Cancer Research, Rhiem and colleagues [1] report contralateral breast cancer risks in relatives of $B R C A 1 / 2$ mutation carriers as well as those testing negative. The authors quote 25 -year risks of $44.1 \%$ for $B R C A 1$ and $33.5 \%$ for $B R C A 2$. The risks quoted are somewhat lower than might be inferred from previous estimates in BRCA1/2 carriers, which have been as high as $40 \%$ at 10 years [2]. This discrepancy may be explained in part by the decision to exclude index cases in which there may have been testing bias to bilateral disease. However, the authors dismiss a second bias of including many non-carriers in their analysis as 'putative' carriers.

Only 319 (16\%) out of 1,909 BRCA1/2 women included in their analysis were proven mutation carriers, and 1,590 relatives were included as first- or second-degree relatives. Interestingly, the authors chose to report the $5 \%$ to $6 \%$ rate of those affected with breast cancer testing negative (phenocopies) from a Dutch prospective analysis as a reason to dismiss the phenocopy rate as having a major effect in their analysis [3]. However, in this Dutch study, of those with just breast cancer, and not ovarian cancer, $11(10 \%)$ out of 113 tested negative [3]. Indeed, the rate in their own paper was 40 (11\%) out of 368 [1]. Given that there was a discrepancy between the 326 carriers in the text and 319 in their table, it is likely that the authors have also included 'obligate' carriers rather than those directly testing mutation-positive. As such, the authors' phenocopy rate is likely higher in untested relatives. In our series of directly tested first-degree relatives, 50 (17.6\%) out of 284 with breast cancer tested negative and 19 (35\%) out of 54 second-degree relatives, and this in line with our previous estimates [4]. The authors do not present the proportion of first-/second-degree relatives in their analysis, so it is difficult to determine how large an effect this could have had. Their assumption that they could include 'clusters' of breast cancer to impute which side of the family a mutation may have come from may add to the phenocopy rate. Often, paternally derived mutations are wrongly inferred, at first, to be maternal because of maternally related breast cancers.

Another potential problem with quoting the rates from the paper to women testing positive for mutations today is that the median years of birth for relatives were 1939 for $B R C A 2$ and 1943 for BRCA1 and median ages at diagnosis of first breast cancer were 43.5 and 48.1 years for $B R C A 1$ and $B R C A 2$, respectively. This would imply a median year of diagnosis of 1987, when breast cancer rates and, presumably, contralateral rates would have been lower [5].

\section{Authors' response}

Rita K Schmutzler, Kerstin Rhiem and Christoph Engel

We thank Evans and colleagues for their valuable comments regarding phenocopies as a potential source of our study. To determine the extent to which phenocopies

*Correspondence: gareth.evans@cmft.nhs.uk

'Genesis Breast Cancer Prevention Centre, University Hospital of South Manchester NHS Trust, Southmoor Road, Wythenshawe, Manchester, M23 9LT, UK

Full list of author information is available at the end of the article could have biased our contralateral breast cancer risk estimates, we performed additional data analyses. The rate of negatively tested breast cancer patients from mutation-positive families in our registry still amounts to $11 \%$ (64 out of 558), which is lower than reported by Evans and colleagues. Among all 6,235 relatives who were analyzed in our study, 4,586 individuals (74\%) were second-degree relatives. Since the largest proportion of phenocopies is expected in the group of second-degree relatives, we excluded this group from the analysis and 
re-calculated the risks based on first-degree relatives only. The 10-year cumulative risks were $25.8 \%$ (95\% confidence interval (CI) $21.5 \%$ to $30.1 \%$ ) for those from BRCA1 families and $15.7 \%$ (95\% CI $10.4 \%$ to $21.0 \%$ ) for those from BRCA2 families. These figures are slightly higher than those based on all relatives. If only relatives with a proven mutation are considered, the 10-year cumulative risks were $28.1 \%$ (95\% CI $19.5 \%$ to $36.7 \%$ ) for BRCA1 carriers and $20.2 \%$ (95\% CI $6.9 \%$ to $33.5 \%$ ) for $B R C A 2$ carriers. Importantly, all of these risks are considerably lower than those obtained from index cases: $38.0 \%$ (95\% CI $33.9 \%$ to $42.1 \%$ ) for BRCA 1 carriers and $22.5 \%$ (95\% CI $17.2 \%$ to $27.8 \%$ ) for $B R C A 2$ carriers.

We agree that quoting risk estimates obtained from retrospective data to women testing positive today holds a number of potential problems. This underlines the urgent need for large and long-term prospective cohort studies to obtain valid risk estimates.

\section{Abbreviation}

$\mathrm{Cl}$, confidence interval.

\section{Competing interests}

The authors declare that they have no competing interests.

\section{Author details}

'Genesis Breast Cancer Prevention Centre, University Hospital of South Manchester NHS Trust, Southmoor Road, Wythenshawe, Manchester, M23 9LT, UK. ${ }^{2}$ Genetic Medicine, The University of Manchester, Manchester Academic Health Science Centre, Central Manchester Foundation Trust, St. Mary's Hospital, Oxford Road, Manchester, M13 9WL, UK. ${ }^{3} \mathrm{NIBHI}$, Centre for Health Informatics, Institute of Population Health, Jean McFarlane Building, The University of Manchester, Oxford Road, Manchester, M13 9PL, UK.
Published: 26 February 2013

\section{References}

1. Rhiem K, Engel C, Graeser M, Zachariae S, Kast K, Kiechle M, Ditsch N, Janni W, Mundhenke C, Golatta M, Varga D, Preisler-Adams S, Heinrich T, Bick U, Gadzicki D, Briest S, Meindl A, Schmutzler RK: The risk of contralateral breast cancer in patients from BRCA1/2 negative high risk families as compared to patients from BRCA1 or BRCA2 positive families: a retrospective cohort study. Breast Cancer Res 2012, 14:R156.

2. Metcalfe K, Lynch HT, Ghadirian P, Tung N, Olivotto I, Warner E, Olopade OI, Eisen A, Weber B, McLennan J, Sun P, Foulkes WD, Narod SA: Contralateral breast cancer in BRCA1 and BRCA2 mutation carriers. J Clin Oncol 2004, 22:2328-2335.

3. Meijers-Heijboer H, Brekelmans CT, Menke-Pluymers M, Seynaeve C, Baalbergen A, Burger C, Crepin E, van den Ouweland AW, van Geel B, Klijn JG: Use of genetic testing and prophylactic mastectomy and oophorectomy in women with breast or ovarian cancer from families with a BRCA1 or BRCA2 mutation. J Clin Oncol 2003, 21:1675-1681.

4. Smith A, Moran A, Boyd MC, Bulman M, Shenton A, Smith L, Iddenden I, Woodward E, Lalloo F, Rahman N, Maher ER, Evans DG: The trouble with phenocopies: are those testing negative for a family BRCA1/2 mutation really at population risk? J Med Genet 2007, 44:10-15.

5. Evans DG, Shenton A, Woodward E, Lalloo F, Howell A, Maher ER: Penetrance estimates for BRCA1 and BRCA2 based on genetic testing in a Clinical Cancer Genetics service setting. BMC Cancer 2008, 8:155. doi:10.1186/bcr3382

Cite this article as: Evans DGR, et al:: Contralateral breast cancer risk in $B R C A 1 / 2$-positive families needs to be adjusted for phenocopy rates particularly in second-degree untested relatives. Breast Cancer Research 2013, 15:401. 